\title{
Paravalvular leakage of transcatheter aortic valve replacements de- pending on aortic annulus calcification
}

Sebastian Kaule, Institute for Implant Technology and Biomaterials e.V., Rostock-Warnemünde, Germany, Alper Oener, Department of Cardiology, University Medical Center Rostock, Germany, Niels Grabow, Institute for Biomedical Engineering, University Medical Center Rostock, Germany

Klaus-Peter Schmitz, Institute for Implant Technology and Biomaterials e.V., Rostock-Warnemünde, Germany, | Institute for Biomedical Engineering, University Medical Center Rostock, Germany

Stefan Siewert, Institute for Implant Technology and Biomaterials e.V., Rostock-Warnemünde, Germany, Michael Stiehm, Institute for Implant Technology and Biomaterials e.V., Rostock-Warnemünde, Germany

\section{Introduction}

Paravalvular leakage (PVL) has a crucial impact on clinical outcomes of transcateheter aortic valve replacements (TAVR), especially the mortality increases dramatically with high-grade PVL. Furthermore, the calcification of the aortic annulus has a decisive influence on the PVL of TAVR. Therefore, we developed a technical model of a calcified aortic annulus (annulus model) and used it for the investigation of PVL in steady-state back-flow conditions.

\section{Methods}

We investigated an Evolut PRO (Medtronic, Minneapolis, MN, USA), implanted the TAVR at different heights ranging between $0 \mathrm{~mm}$ and $-6 \mathrm{~mm}$ and characterized PVL in steady-state retrograde flow from $0 \mathrm{mmHg}$ up to a maximum achievable pressure. The used test bench and detailed test method was described in previous studies. The annulus model exhibits three elevations symmetrically distributed around the circumference. Depending on the degree of calcification the elevations reach into the lumen from $1 \mathrm{~mm}$ to $3 \mathrm{~mm}$.

\section{Results}

For the Evolut PRO, a decreasing PVL was measured with increasing implantation depth. At an implantation depth of $0 \mathrm{~mm}$ (inflow of TAVR and annulus model at same height) maximum PVL was measured. Minimum PVL was found at a height of $-6 \mathrm{~mm}$. Furthermore, even a small calcification of $1 \mathrm{~mm}$ led to a large increase in PVL. This trend continued with increasing height of the calcification. The maximum regurgitation of $(2,025.21 \pm 12.47) \mathrm{ml}(n=3$ measurements $)$ was measured at a pressure of $6 \mathrm{mmHg}$ in the annulus model with $3 \mathrm{~mm}$ calcification.

\section{Conclusion}

A test method to quantify PVL depending on annular calcification was successfully developed. Additionally, the influence of implantation height on PVL was characterized. Due to the technical operating principle of the test bench, only a limited increase in pressure was possible, depending on the amount of retrograde volume flow. In this respect, the test rig must be optimized in the future. 


\title{
Systematic morphological characterization of sub-visible particles generated from cardiovascular devices using dynamic image analysis
}

\author{
Anja Kurzhals, Institute for Biomedical Engineering, Rostock University Medical Center, Rostock-Warnemuende, \\ Germany, anja.kurzhals@uni-rostock.de \\ Christoph Brandt-Wunderlich, Institute for ImplantTechnology and Biomaterials e.V, Rostock-Warnemuende, Germa- \\ ny, christoph.brandt-wunderlich@uni-rostock.de \\ Klaus-Peter Schmitz: Institute for ImplantTechnology and Biomaterials e.V. and Institute for Biomedical Engineering, \\ Rostock University Medical Center, Rostock-Warnemuende, Germany, klaus-peter.schmitz@uni-rostock.de \\ Niels Grabow, Institute for Biomedical Engineering, Rostock University Medical Center, Rostock-Warnemuende, Ger- \\ many, niels.grabow@uni-rostock.de \\ Wolfram Schmidt, Institute for Biomedical Engineering, Rostock University Medical Center, Rostock-Warnemuende, \\ Germany, wolfram.schmidt@uni-rostock.de
}

\section{Introduction}

The assessment of particulate matter generated during a simulated use is requested by international standards for approval of cardiovascular devices. Within the new revision of ISO 25539-2:2020 in addition to a quantitative evaluation of particulate matter, a morphological characterization is required to gain information about the particles origin. Within this study the dynamic image analysis (DIA) was used in order to establish a systematic morphological classification of particles.

\section{Methods}

The particle suspension was generated during a simulated use test procedure of a commercially available coronary stent delivery system (delivery, deployment and retraction within a vascular model). It was analyzed by DIA using the FlowCam 8000 (Yokogawa Fluid Imaging Technologies Inc., USA): A $5 \mathrm{ml}$ aliquot of the particle suspension was directed through a $300 \mu \mathrm{m}$ flow cell, where images of each particle were generated with the help of a 4x lens (OLYMPUS, Japan). The particle images were evaluated concerning several properties such as equivalent spherical diameter (ESD), circularity, aspect ratio and intensity.

\section{Results}

The DIA revealed a total of 858 evaluable particle images of relevant particles $\geq 10 \mu \mathrm{m}$ (ESD), whereas $53 \%$ were larger than $25 \mu \mathrm{m}$ (ESD). The particle shapes were inhomogenous resulting in a wide ranging aspect ratio of 0.03 to 0.95 . The circularity decreased with increasing diameter. Around $83 \%$ of the particles had an intensity value larger than 140 grey scale units. These particles appeared translucent and might supposely origin from the lubricious coating of the guide wires or catheters.

\section{Conclusion}

The DIA appears suitable for a morphological analysis of particle suspensions and can gain information for the determination of the particles origin. For an effective particle assessment the DIA analysis parameters should be selected depending on the particular scientific problem. The evaluation of the particle properties should be supported by results of more methods (e.g. SEM, Raman spectroscopy). 


\section{In vitro biocompatibility testing of polymeric nanofiber scaffolds: fine- tuning for a better prediction of the in vivo behaviour}

Valeria Khaimov (Institute for ImplantTechnology and Biomaterials e.V.), Rostock-Warnemünde, Germany, valeria.khaimov@uni-rostock.de,

Bastian-Jesper Klußmann-Fricke (Institute for ImplantTechnology and Biomaterials e.V. and Institute for Biomedical Engineering, Rostock University Medical Center), Rostock-Warnemünde, Germany, bastian.klussmann-fricke@unirostock.de

Sabine Illner (Institute for Biomedical Engineering, Rostock University Medical Center), Rostock-Warnemünde, Germany, sabine.illner@uni-rostock.de

Stefan Siewert, Institute for ImplantTechnology and Biomaterials e.V, Rostock-Warnemuende, Germany, stefan.siewert@uni-rostock.de

Klaus-Peter Schmitz (Institute for ImplantTechnology and Biomaterials e.V. and Institute for Biomedical Engineering, Rostock University Medical Center), Rostock-Warnemünde, Germany, klaus-peter.schmitz@iib-ev.de

\section{Introduction}

Biomaterial research efforts focus on the development of biomaterials mimicking the natural extracellular environment. In addition, materials used in blood-contacting medical devices should minimize activation of platelets, the coagulation cascade and complement system. A confluent thin layer of endothelial cells lines all blood vessels and produces factors responsible for inhibition of coagulation, thrombosis and neointimal hyperplasia. Thus, the ability to rapidly form a healthy endothelium upon implantation represents a desired property of biomaterials used for cardiovascular devices. In this study we used advanced in vitro methods to investigate the biocompatibility of a permanent and a biodegradable electrospun nanofiber fabric, poly-L-lactic acid and polycarbonate-based silicone elastomers respectively, with the focus on endothelization and hemocompatibility.

\section{Methods}

Evaluation of nonwovens was performed in agreement with DIN EN ISO 10993. Cytotoxicity was assessed by measuring viability of human endothelial cells upon treatment with extracts for 48 hours. Endothelialization potential was assessed by seeding cells directly onto the scaffolds followed by viability measurement and quantitative microscopic analysis. For hemocompatibility testing electrospun scaffolds were analyzed by ELISA and/or scanning electron microscopy upon contact with either platelet-rich plasma or human serum.

\section{Results}

Non of the materials was cytotoxic. Microscopy analysis revealed poor cell attachment and morphology of endothelial cells grown directly on the biomaterial surface using the standard protocol. Cell adhesion partially improved when scaffolds were treated by vacuum degassing, serum or plasma prior to seeding. in vitro hemocompatibility testing revealed no activation of the complement system as well as moderate platelet activation.

\section{Conclusion}

Besides low cytoxicity, formation of a functional endothelial layer is crucial for the performance of blood-contacting implants. Our data shows that fine-tuning of a commonly used in vitro testing systems can have a huge impact on the performance of biomaterials. Moreover, our in vitro complement activation assay provides a robust tool for initial screening and optimization of biomaterials with respect to hemocompatibility. 


\section{Automation of online particle measurement during the simulated use of catheters and stent systems}

Inga Wiese, Institute for Biomedical Engineering, Rostock University Medical Center, Rostock-Warnemuende, Germany, inga.wiese@uni-rostock.de

Anja Kurzhals, Institute for Biomedical Engineering, Rostock University Medical Center, Rostock-

Warnemuende, Germany, anja.kurzhals@uni-rostock.de

Grit Rhinow, Institute for Biomedical Engineering, Rostock University Medical Center, Rostock-Warnemuende, Germany, grit.rhinow@uni-rostock.de

Carsten Tautorat, Institute for Biomedical Engineering, Rostock University Medical Center, Rostock-

Warnemuende, Germany, carsten.tautorat@uni-rostock.de

Frank Kamke, Institute for ImplantTechnology and Biomaterials e.V., Rostock-Warnemuende, Germany,

frank.kamke@iib-ev.de

Christoph Brandt-Wunderlich, Institute for ImplantTechnology and Biomaterials e.V., Rostock-Warnemuende, Germany, christoph.brandt@iib-ev.de

Klaus-Peter Schmitz: Institute for ImplantTechnology and Biomaterials e.V. and Institute for Biomedical Engineering, Rostock University Medical Center, Rostock-Warnemuende, Germany, klaus-peter.schmitz@unirostock.de

Niels Grabow, Institute for Biomedical Engineering, Rostock University Medical Center, Rostock-

Warnemuende, Germany, niels.grabow@uni-rostock.de

Wolfram Schmidt, Institute for Biomedical Engineering, Rostock University Medical Center, RostockWarnemuende, Germany, wolfram.schmidt@uni-rostock.de

\section{Introduction}

The assessment of the coating integrity of cardiovascular implants, such as catheters and stent systems, is of crucial importance for their approval and is required by international standards (ISO, ASTM), as well as FDA guidance documents. Within this study a test bench for online particle measurements during the simulated use of catheters and stent systems was automated to optimize the reproducibility of test results.

\section{Methods}

Electronically controlled valves and a central processing unit were added to the test bench. Using the graphical programming interface Node-RED, the data communication between the sensors and the valves as well as the data storage of the particle count and volume flow data was implemented. With the automated test routine, measurements of standard particles with sizes of $10 \mu \mathrm{m}, 25 \mu \mathrm{m}$ and $50 \mu \mathrm{m}$ were determined.

\section{Results}

With the automated control of the valves the volume flow range could be maintained as required for correct particle detection $(75 \mathrm{ml} / \mathrm{min})$. The recovery rate of the number of particles in size classes $25 \mu \mathrm{m}$ and $50 \mu \mathrm{m}$ was $98.3 \pm 0.9 \%$ and $96.6 \pm 2.2 \%$ which corresponds to the requirements of the FDA. The recovery rate of the $10 \mu \mathrm{m}$ particles was $85.7 \pm 1.6 \%$ and is thus $4.3 \%$ below the recovery rate of $90 \%$ required by the FDA. However a comparison with the test results generated using the manual test routine shows that automation decreases the scattering of the data between individual measurements. The standard deviations while using the manual test routine were $\pm 3.7 \%(10 \mu \mathrm{m}), \pm 10.2 \%(25 \mu \mathrm{m})$ and $\pm 17.2 \%(50 \mu \mathrm{m})$.

\section{Conclusion}

The automation of the test bench for online particle measurements could be performed successfully. The handling for users was simplified. Recovery testing with particle count standards shows very promising results but require further investigations. However, the reproducibility of the measurements could be increased compared to the manual test routine. 


\section{Particle Image Velocimetry for in vitro characterization of Paravalvular Leakage of TAVR-sealing concepts}

Samuel Höing, Institute for ImplantatTechnology and Biomaterials e. V., Rostock-Warnemünde, Germany, samuel.hoeing@uni-rostock.de

Finja Borowski, Institute for ImplantatTechnology and Biomaterials e. V., Rostock-Warnemünde, Germany, finja.borowski@uni-rostock.de

Jan Oldenburg, Institute for ImplantatTechnology and Biomaterials e. V., Rostock-Warnemünde, Germany, jan.oldenburg@uni-rostock.de

Sabine Illner, Institute for Biomedical Engineering, Rostock University Medical Center, Rostock-Warnemünde, Germany, sabine.illner@uni-rostock.de

Alper Öner, Department of Cardiology Rostock University Medical Center, Rostock, Germany, alper.oener@med.uni-rostock.de

Michael Stiehm, Institute for ImplantatTechnology and Biomaterials e. V., Rostock-Warnemünde, Germany, michael.stiehm@uni-rostock.de

Klaus-Peter Schmitz, Institute for ImplantatTechnology and Biomaterials e. V., Rostock-Warnemünde, Germany, schmitz@iib-ev.de

Sebastian Kaule, Institute for ImplantatTechnology and Biomaterials e. V., Rostock-Warnemünde, Germany, sebastian.kaule@uni-rostock.de

\section{Introduction}

Paravalvular leakage (PVL), defined as the leakage between the aortic annulus and a transcatheter aortic valve replacement (TAVR), is verifiably associated with long-term clinical outcome, especially with increased mortality. Therefore, with the ambition to reduce or even prevent PVL of next generation TAVR, it is necessary to extend the hemodynamic understanding of PVL.

This study presents an in vitro flow measurement method to localize PVL during hydrodynamic characterization of TAVR and furthermore presents different design features, so-called TAVR-skirt, to reduce PVL.

\section{Methods}

Particle image velocimetry (PIV) measurements were performed for flow field assessment during hydrodynamic characterization of TAVR. Additionally, two different skirt-designs were developed to reduce PVL. The skirts were manufactured from polymeric-nonwoven and sued to pericardium-based TAVR-prototype.

The prepared TAVR-prototype was then deployed in a pathophysiological model of the aortic root with a calcification nodule of $2 \mathrm{~mm}$ according to ISO 5840:2021. To assess PVL, the flow field and the regurgitation volume was measured.

\section{Results}

The PIV measurements showed a clearly visible leakage jet between the TAVR-prototypes without skirt and the pathophysiological aortic annulus model. Jet velocities of up to $0.5 \mathrm{~m} / \mathrm{s}$ were measured depending on presence or configuration of a PVL-preventing skirt. When implanted in the physiological annulus model without calcification nodule, PVL was hardly recognizable.

The regurgitation volume of a TAVR-prototype without skirt at $51 / \mathrm{min}$ was $36.26 \pm 1.89 \mathrm{ml}(n=10)$. The developed and manufactured polymeric-nonwoven skirts reduced PVL from $37.67 \pm 1.17 \mathrm{ml}$ to $18.36 \pm 1.8 \mathrm{ml}$ ( $n=10$, TAVR-skirt-design1) and from $46.97 \pm 1.07 \mathrm{ml}$ to $17.85 \pm 1.29 \mathrm{ml}(n=10$, TAVR-skirt-design2) at $5 \mathrm{l} / \mathrm{min}$.

\section{Conclusion}

The localization of PVL during hydrodynamic characterization by means of PIV was successful. The sealing concepts developed in this work were very effective and led to a PVL-reduction of the tested TAVP prototypes of about $50 \%$ to $60 \%$. 


\title{
Validation of a Fluid-Structure Interaction Model for the Characterization of Transcatheter Mitral Valve Repair Devices
}

\author{
Robert Ott: Institute for ImplantTechnology and Biomaterials e.V., Friedrich-Barnewitz-Str. 4, 18119 Rostock- \\ Warnemünde, Germany, E-mail: robert.ott@uni-rostock.de \\ Alper Öner: Heart Center/Department of Cardiology, Rostock University Medical Center, 18057 Rostock, Germany, \\ E-mail: alper.oener@med.uni-rostock.de \\ Paul Hermann Bellé: Institute for ImplantTechnology and Biomaterials e.V., Friedrich-Barnewitz-Str. 4, 18119 \\ Rostock-Warnemünde, Germany, E-mail: paulbelle@outlook.de \\ Finja Borowski: Institute for ImplantTechnology and Biomaterials e.V., Friedrich-Barnewitz-Str. 4, 18119 Rostock- \\ Warnemünde, Germany, E-mail: finja.borowski@uni-rostock.de \\ Klaus-Peter Schmitz: Institute for ImplantTechnology and Biomaterials e.V., Friedrich-Barnewitz-Str. 4, 18119 \\ Rostock-Warnemünde, Germany, E-mail: klaus-peter.schmitz@uni-rostock.de \\ Michael Stiehm: Institute for ImplantTechnology and Biomaterials e.V., Friedrich-Barnewitz-Str. 4, 18119 Rostock- \\ Warnemünde, Germany, E-mail: michael.stiehm@uni-rostock.de
}

\section{Introduction}

Mitral regurgitation (MR) is the second most frequent indication for heart valve surgery and catheter interventions. According to European and US-American guidelines, transcatheter mitral valve repair (TMVR) maybe be considered a treatment option for selected high-risk patients. However, the biomechanical impact of TMVR-devices on the mitral valve (MV) has not yet been fully understood. In order to address this problem, a 3D-Fluid-Structure Interaction (FSI) framework utilizing non-linear Finite Element Analysis for the MV and Smoothed Particle Hydrodynamics for the fluid flow was developed and validated against in vitro data.

\section{Methods}

An artificial MV-model (MVM) with a prolapse in the A2-P2 region and a custom-made TMVR-device implanted in the A2-P2 region were used for the in vitro investigations. In accordance with ISO 5910, projected mitral orifice areas (PMOA), flow rates as well as atrial and ventricular pressures were measured under pulsatile flow conditions before and after TMVRdevice implantation. For the FSI-model, the MVM-geometry was reconstructed by means of micro-computed tomography in a quasi-stress-free configuration. Quasi-static tensile test data was utilized for the development of linear- and hyperelastic material models of the chordae tendinae and leaflets, respectively. The fluid flow was modelled assuming an incompressible, homogenous Newtonian behaviour. Time-varying in vitro atrial and ventricular pressure loading was applied as boundary condition for two cardiac cycles for both the pre- and post-TMVR-device implantation model.

\section{Results}

In vitro investigations show that TMVR-device implantation in the A2-P2 region effectively reduces the regurgitation fraction (RF) from $48 \%$ to $18 \%$. Moreover, the minimum PMOA during systole decreases from $0.13 \mathrm{~cm}^{2}$ to zero, and the maximum PMOA during diastole decreases from $2.40 \mathrm{~cm}^{2}$ to $1.02 \mathrm{~cm}^{2}$. The comparison of experimental and numerical data yields a deviation of $2.8 \%$ for the RF and a maximum PMOA-deviation of $3.5 \%$ for both the pre- and post-implantation-state.

\section{Conclusion}

The developed FSI-framework is in good agreement with in vitro data and is applicable for the characterization of the biomechanical impact of different TMVR-devices under varying flow conditions. 